\title{
An Expanding Heart: A Case of a Left Ventricular Rupture
}

\author{
Stephen Huelskamp, MD and Jessica Dahmus, MD
}

\section{INTRODUCTION}

A ventricular aneurysm was once a common complication of a myocardial infarction (MI), seen in up to 35 percent of coronary occlusion with transmural tissue damage. ${ }^{1}$ The advent of percutaneous coronary intervention (PCI) and thrombolytic therapy, which produced effective arterial patency, had a direct correlation with the reduction in the number of myocardial infarctions complicated by aneurysm, with the current incidence at 15 percent. $^{2}$ Aneurysmal formation and rupture is a life-threatening complication of an $\mathrm{Ml}$ and clinicians should be aware of its signs and management. We present a case of ST elevation MI (STEMI) complicated by left ventricular aneurysmal formation and rupture post PCl.

\section{CASE PRESENTATION}

A 75-year-old male with a history of hypertension, hyperlipidemia, type 2 diabetes mellitus, benign prostatic hypertrophy, and recent STEMI status post bare metal stent to the distal right coronary artery (RCA) re-presented five days post MI with substernal chest and back pain. His exam was significant for mild distress and a friction rub. Electrocardiogram (EKG) showed 3-5 mm inferior and lateral ST elevations. Troponin T was 1.29. Emergent left heart catheterization showed no change from previous intervention. Transthoracic echocardiography (TTE) at this time was unchanged from previous hospitalization, consistent with left ventricular dysfunction, akinesis of several walls, and unchanged left ventricular aneurysm without evidence of rupture or pericardial effusion. Given the continued chest pain, elevated troponin, and new pericardial friction rub, cardiac CT was performed to evaluate the myocardium which showed a markedly thinned aneurysmal wall without signs of rupture. Although aneurysmorrhaphy was considered, the patient was deemed a poor surgical candidate due to age and poor functional capacity.

\section{DIFFERENTIAL DIAGNOSIS}

Post transmural $\mathrm{MI}$ complications are becoming less frequent due to improvements in medical management and early intervention. Recurrent chest pain post STEMI has a broad differential including aneurysm, pseudoan- eurysm, Takotsubo cardiomyopathy, stent thrombosis, papillary muscle rupture, septal wall rupture, pericarditis, ventricular wall rupture, and repeat acute coronary syndrome. Physical examination findings such as a pericardial friction rub and EKG findings such as ST elevation of the anterior leads or diffuse ST-segment elevation in the setting of recent $\mathrm{Ml}$ can raise suspicion of aneurysmal formation. Imaging with TTE, cardiac computerized tomography, or MRI can confirm the diagnosis.

\section{OUTCOME AND FOLLOW UP}

Within one hour of returning from CT, the patient developed dyspnea, wheezing, and PEA cardiac arrest. Bedside TTE during the cardiac arrest revealed myocardial rupture with hematoma in the pericardial sac compressing the right ventricle consistent with cardiac tamponade. The family decided to stop resuscitative measures, and the patient expired.

\section{DISCUSSION}

Myocardial rupture is a rare complication of left ventricular aneurysms and is more common in pseudoaneurysms. A true aneurysm is a thinned, scarred, or fibrotic distention of the ventricular wall devoid of muscle due to transmural infarction while a pseudoaneurysm is a free wall rupture that is contained by localized pericardial adhesions. The diagnosis of a true aneurysm is difficult but has become more readily identified through increased precision of imaging techniques. Echocardiogram is typically the first imaging modality utilized but is less specific in identifying true aneurysm versus pseudoaneurysm. Left ventricular angiography is the most reliable diagnostic technique, followed by cardiac CT or MRI. ${ }^{3}$ Early differentiation between true aneurysm versus pseudoaneurysm can aid in determining the most appropriate treatment strategy. ${ }^{4}$ Small to moderate true aneurysms are more likely to respond to medical therapy through the use of afterload reduction, with elective surgical intervention as an option for larger and less responsive aneurysms. Untreated pseudoaneurysms have a 30-45\% risk of rupture, making surgical intervention with aneurysmorrhaphy a necessary procedure. ${ }^{5}$ 


\section{KEY POINTS}

Although an aneurysm or pseudoaneurysm is not the only or most frequent structural complication post-MI, myocardial aneurysmal expansion can be a devastating and potentially lethal complication. Due to early and effective treatments of $\mathrm{MI}$, aneurysmal formations are less frequent. ${ }^{6}$ Clinicians should have a high suspicion for this complication if a patient presents with pain, elevated troponin, diffuse ST-segment elevations, and friction rub after a recent myocardial infarction and should be prepared for the necessity of rapid surgical or palliative management.

\section{REFERENCES}

1. Mills NL, Everson CT, Hockmuth DR. Technical advances in the treatment of left ventricular aneurysm. Ann Thorac Surg. 1993;55(3):792-800.

2. Tikiz H, Balbay Y, Atak R, Terzi T, Genç Y, Kütük E. The effect of thrombolytic therapy on left ventricular aneurysm formation in acute myocardial infarction: relationship to successful reperfusion and vessel patency. Clin Cardiol. 2001;24(10):656-62

3. Al-Saadon K, Walley VM, Green M, Beanlands DS. Angiographic diagnosis of true and false LV aneurysms after inferior wall myocardial infarction. Cathet Cardiovasc Diagn; 1995; 35(3): 266.

4. Zoffoli G, Mangino D, Venturini A, et al. Diagnosing left ventricular aneurysm from pseudo-aneurysm: a case report and a review in literature. $J$ Cardiothorac Surg. 2009:4:11

5. Vlodaver Z, Coe JI, Edwards JE. True and false left ventricular aneurysms Propensity for the latter to rupture. Circulation. 1975; 51(3): 567.

6. Visser CA, Kan G, Meltzer RS, Koolen JJ, Dunning AJ. Incidence, timing and prognostic value of left ventricular aneurysm formation after myocardial infarction: a prospective, serial echocardiographic study of 158 patients. Am J Cardiol. 1986:57(10):729-32.

7. Dubnow MH, Burchell HB, Titus JL. Postinfarction ventricular aneurysm. A clinicomorphologic and electrocardiographic study of 80 cases. Am Heart J. 1965;70(6):753-60.

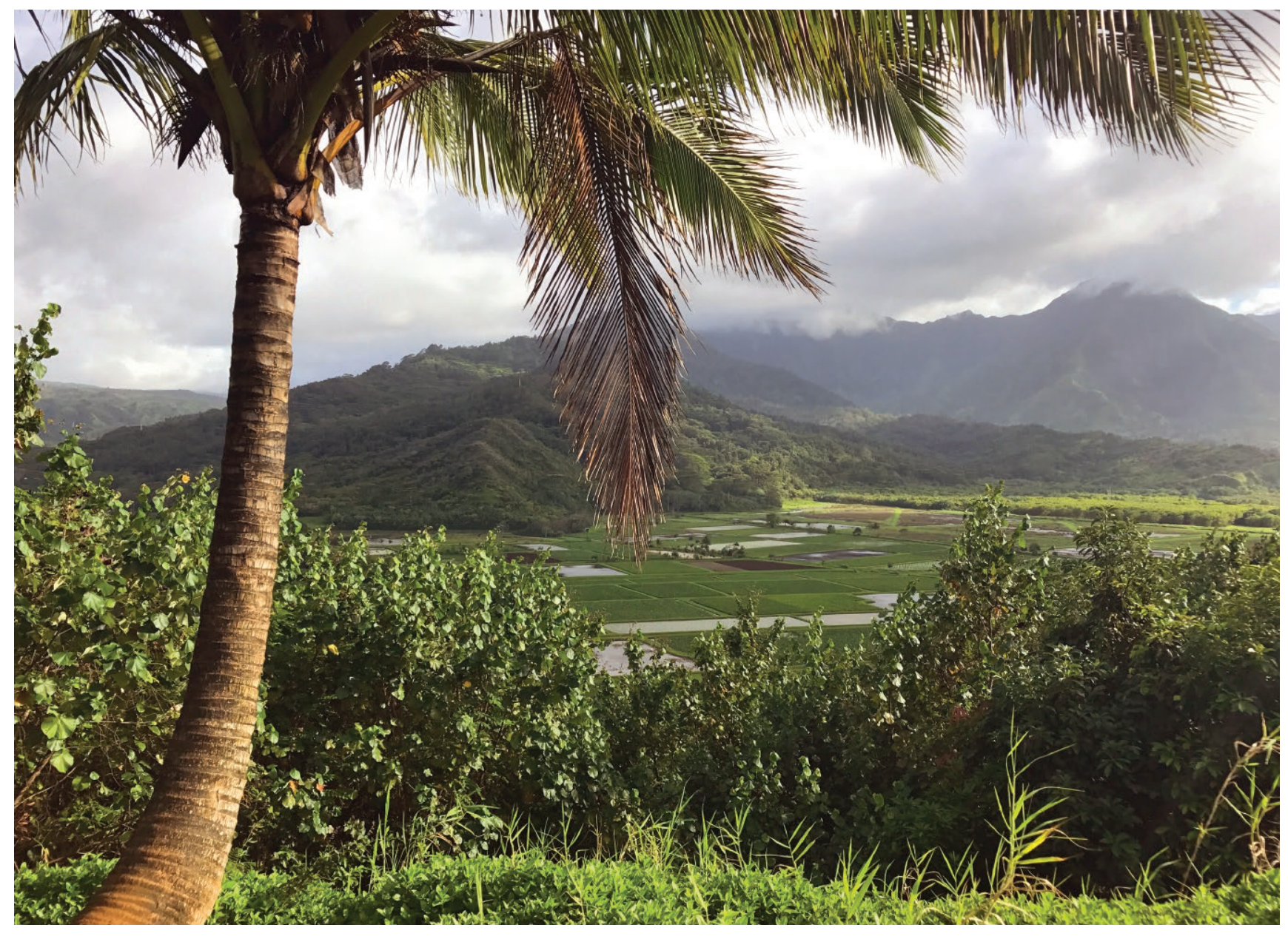

Shuwei Wang, MD 